\title{
FUSION AS A FUTURE ENERGY SOURCE
}

D.J. Ward - CCFE, Culham Science Centre, Abingdon, OX14 3DB, UK. - DOI: http://dx.doi.org/10.1051/epn/2016505

\author{
Fusion remains the main source of energy generation in the Universe and is \\ indirectly the origin of nearly all terrestrial energy (including fossil fuels) but it is \\ the only fundamental energy source not used directly on Earth. Here we look at the \\ characteristics of Earth-based fusion power, how it might contribute to future energy \\ supply and what that tells us about the future direction of the R\&D programme. \\ The focus here is Magnetic Confinement Fusion although many of the points apply \\ equally to inertial confinement fusion.
}

\section{Fusion Characteristics as an Energy Source}

\section{Resources}

The potential energy resource from fusion is enormous, primarily because the energy produced per unit mass of fuel is so large, and this is the principal reason why fusion $R \& D$ has been pursued. If we were able to harness energy simply from the fusion of two deuterium atoms (D-D fusion), which is possible in theory but difficult in practise, we could supply mankind's energy needs for billions of years. At present, however, we have a much less ambitious target, the fusion of deuterium with tritium (D-T fusion), which is roughly 100 times higher in cross-section. As tritium decays with a half-life of 12.3 years, it is not abundant on Earth so the plan is for a fusion power plant to be self-sufficient in tritium, using the neutrons which result from the fusion reaction to convert lithium into tritium. With present estimated fuel reserves of lithium, there is enough to provide mankind's energy needs for thousands of years, by which time we may have solved the problem of D-D fusion.

Designing a fusion plant to be self-sufficient in tritium is not a trivial problem. Although simply surrounding a fusion neutron source with natural lithium can, in theory, produce up to $70 \%$ more tritium than required, the practical reality is more difficult [1]. There needs to be a box structure to

\footnotetext{
- FIG. 1:

Calculated tempe-

ratures in ITER

components in the

event of a full loss of

cooling water, taken

from [6] (the curve

marked VV illustrates

the temperature at

the Vacuum Vessel).
}

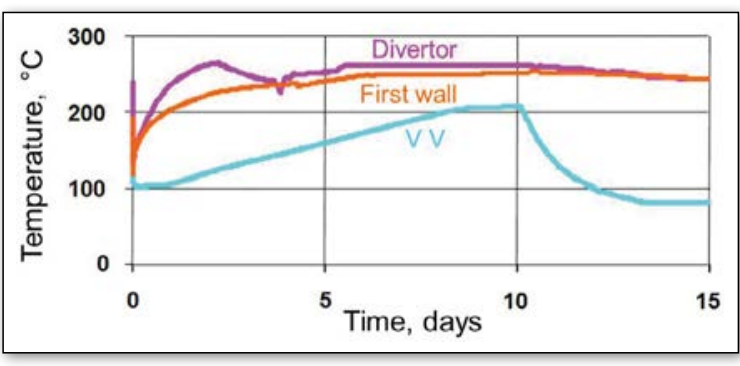

contain the lithium compound, there needs to be coolant flowing to take away the large amounts of energy deposited by the neutrons and we want to restrict the thickness of the structure to minimise the size of the containing magnets. In existing designs, changing the isotopic mix of the lithium and including a neutron multiplier such as beryllium or lead are used as tools to produce an optimised design, but this is far from complete and demonstrated. Tests of these fusion blankets are proposed for the ITER device.

One aspect of tritium supply will relate to the growth phase of fusion power. To start up a new power plant, an initial inventory of tritium is required and this will have to come either from another fusion plant or from a fission plant. Particularly in the early implementation phase, it is important to minimise the inventory of tritium needed to start up a new plant whilst ensuring sufficient tritium is available.

\section{Waste}

One of the differentiating factors of fusion when compared to fission is the waste products. Unlike fission, fusion reactions do not produce radioactive waste directly but the neutrons can cause radioactivity in the surrounding materials - hence the emphasis on developing and testing of low activation materials, those that do not generate long-lived radioactive waste [2]. The same happens in fission plants of course but there the structural activation is a very small part of the waste produced - in fusion it is the dominant part. One goal of the fusion $\mathrm{R} \& \mathrm{D}$ programme is to optimise the use of materials, for instance to minimise the need for repository storage, perhaps to zero, but this is not guaranteed. Optimising material properties is a key, and somewhat neglected area, and would be substantially advanced by a major materials test programme, including a source of fusion relevant, $14 \mathrm{MeV}$ neutrons, such as the proposed IFMIF [3], as this is not an area of particular focus for ITER. 


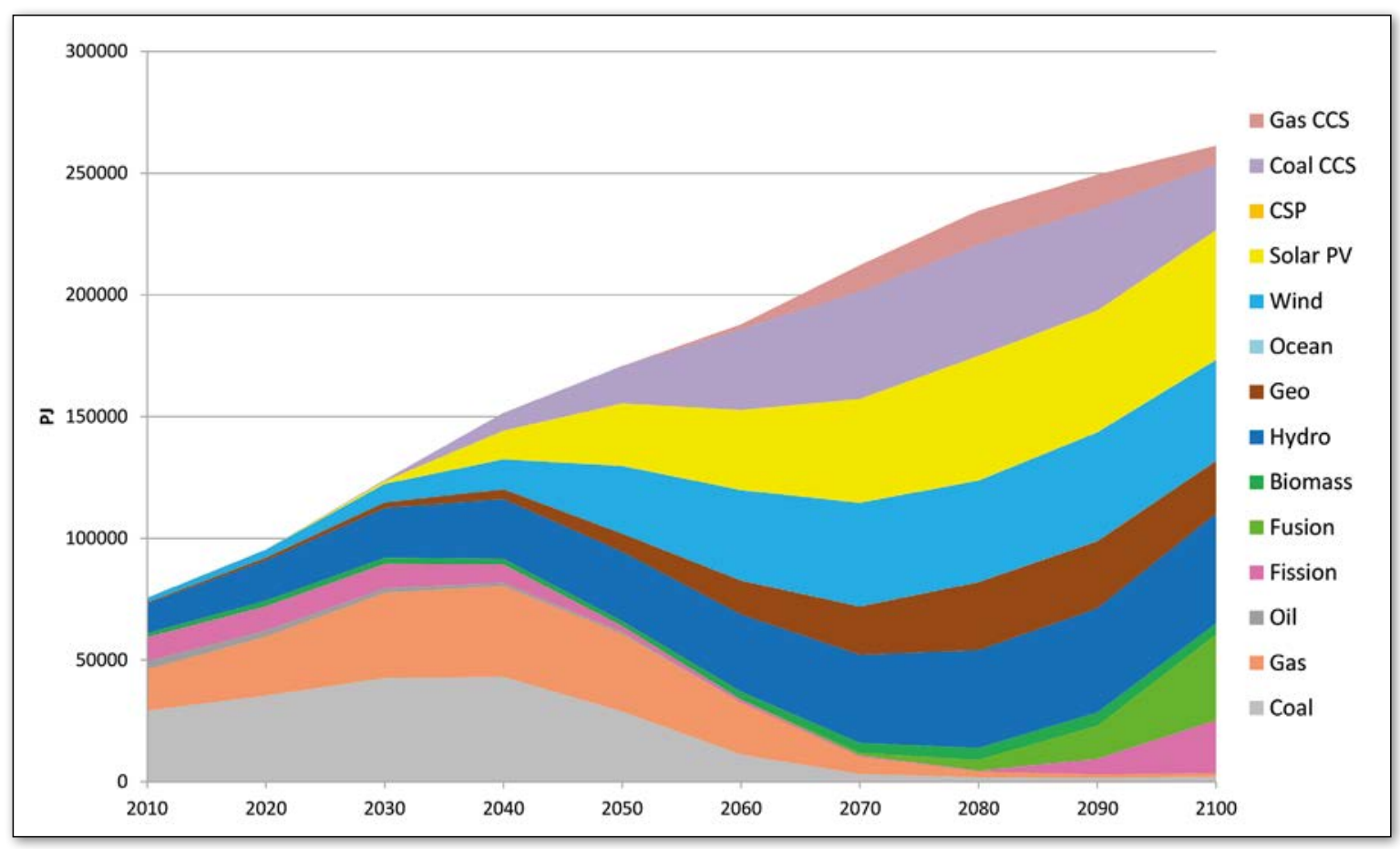

\section{Emissions}

Apart from the large potential resource the main benefit of fusion as foreseen is the lack of carbon emissions. Because it does not rely on combustion of fuels, fusion is intrinsically a low carbon energy source and the atmospheric emissions of other pollutants such as particulates, $\mathrm{NO}_{\mathrm{x}}$ etc are also low. Evaluating the externalities of different energy sources, including fusion, leads to the conclusion that combustion-based technologies generally have the highest externalities and that the external costs of fusion are low, primarily because of low atmospheric emissions $[4,5]$.

\section{Safety}

One aspect of fusion plant design that is considered more important than others, when optimising design, is safety. Because of the low energy available to drive an accident and the low hazard to be released in the event of an accident, fusion plants are proposed as passively safe, that is they should not cause substantial damage even if all active safety systems fail (for instance see Fig. 1). In generic studies, the objective has been to design a plant in which no design-basis accident requires the evacuation of the local population. In working with specific devices in specific locations this has to be refined to the local conditions, for instance a lot of work has been done on the safety of ITER [6]. As with tritium production, this is an active area of research for future power plants, informed by the ongoing work for the design and construction of ITER.

\section{Costs}

An area of substantial uncertainty relates to future costs of fusion power plants. Given the incomplete information for designing a power plant it is difficult to be precise about costs, although the main cost items are already known, particularly buildings, magnets and conventional equipment. In the conceptual designs that have been investigated in the past targets such as generating electricity at less than $0.1 € / \mathrm{kWh}$ have been used as a guide [4]. There are two particular uncertainties presently: information emerging from ITER is providing extra information on the costs of fusion, and the assumptions about plant availability in spite of needing to regularly change components in a power plant are known to be challenging. At the same time the future costs of other energy systems are also very uncertain so it is difficult to be conclusive in cost assessments - nonetheless, cost should be a strong influencing factor in design optimisation. Again, ITER is providing key information in this area.

\section{Fusion in the Future Energy Market}

In discussing fusion's role in a future energy market there are a number of key questions:

- How large will future demand for energy, particularly low carbon energy, be?

- Will fusion be available, when and at what cost relative to other low carbon sources?

- How will public acceptance of different energy technologies evolve and will that increase or reduce fusion's potential role?

The course appears to be set for emerging economies to become significant users of energy, substantially larger than present demand in developed economies; at present the pressure for this to come from low carbon sources is strong and increasing. This presents a future of dramatically increasing challenge; meeting carbon emissions targets in 2030 for instance does little to contribute to meeting targets foreseen for 2070, by which time global energy demand may have doubled again but allowable emissions fallen by another factor of 4 .
FIG. 2: Example of an exploration of how future electricity demand may be met, in a future of reduced carbon emissions. Taken from [8]. 
The route to fusion power was originally addressed in the 1970's, at which point the work needed to achieve fusion as an energy source was mapped out and the cost of doing that work estimated. In present money that would exceed $\$ 50 \mathrm{~B}$ [7].JET has used about $2 \%$ of the resources that were identified as necessary at that time; ITER is likely to take us around $30-40 \%$ of the remaining way. The additional work is focussed on materials and technology testing leading to the construction of a demonstration (DEMO) device.

In costs, superconducting magnets are very expensive today but costs will fall. Fusion has strong economies of scale so larger plants are expected to be more cost competitive. The comparators for fusion may be advanced fission, solar with energy storage and coal with (improved) Carbon Capture and Storage, but such comparisons cannot be made reliably today. Given that developing a new energy source, fusion, as a low carbon energy option can be done with a tiny fraction of the resources needed to transform global energy systems to low carbon (at less than $0.1 \%$ of investment in other energy systems over the same period) it would be imprudent to stop that development on the basis of cost alone.

The influence of future public opinion is difficult to predict. In some world regions the present view of risks from radiation is very distorted when compared to other, much larger, risks to human health such as air pollution or transport accidents, and if this continues, in some world regions at least, then advanced nuclear fission plants may be disfavoured and fusion more likely to play a role. This is something that must continue to be an important consideration in the fusion R\&D programme, influencing the work to optimise a fusion plant in terms of cost, safety, emissions and waste.

\section{An evolving energy system}

We have seen how the different properties of fusion make for a complex optimisation when designing a power plant and how the present programme, particularly ITER, contributes to exploring this. This same complexity extends to designing an optimised energy supply system and the role that fusion can play within it.

There is a big question around the speed with which an energy system can be changed. If a new energy technology became available in 2050, for instance, how long would it be before it could be a major player on the future energy scene $[8,9]$ ? A limit to the growth rate of a new technology may be as high as $25 \%$ per year or as low as $10 \%$ and this could have a significant impact on the introduction of fusion. Assuming that a target of $1 \mathrm{TW}$ installed power is needed for a significant contribution to future energy supply, then the initial scale of introduction and the growth rate determine how quickly this could be achieved. If 20 countries each introduced a $1 \mathrm{GW}$ plant in 2050 (and there were enough tritium to start them up) then a growth to 1TW would take between 17 and 40 years, depending on the growth rate. Although this is not a major issue today, it is an example of how consideration of the "market pull" can feedback to plant requirements, design and hence the R\&D programme. Figure 2 shows an example of attempts to look at future energy scenarios, including fusion, particularly as fusion goes through a growth phase.

\section{Impact on the R\&D Programme}

Producing an optimised working fusion power station is the goal of the European fusion R\&D programme. Optimisation includes designing for cost, availability, safety, emissions, waste, growth rate etc. and is a non-trivial problem, not least because the different areas can pull in different directions and because the energy systems in which fusion will be embedded differ around the world. As the R\&D moves away from physics towards technology, these new areas become increasingly important and a new skill set is required; fusion is moving out of the laboratory and the expertise contained within the R\&D programme must develop accordingly.

\section{About the Author}

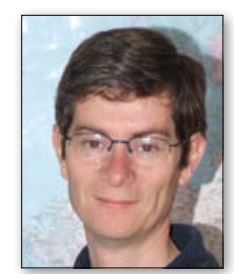

David Ward has worked in fusion since the first JET plasma and had strong involvement in the first JET deuterium-tritium experiments, in which more than $1 \mathrm{MW}$ of controlled fusion power was first produced on Earth, and the later experiments in which up to $16 \mathrm{MW}$ of fusion power was produced. After many years of working on both theory and experiment, David took on the role of leading the JET work carried out to help in the design of the ITER. From there the transition to technical work and a management role in systems studies for power plants, in particular DEMO, was a natural step.

The work in integrated design of a conceptual power plant includes determining the expected properties of fusion as a power source with a natural link to other energy systems and the likely role of fusion in a future energy market. This has involved collaborations with other energy researchers, outside fusion and also led to David's selection as the EUROfusion Project Leader for fusion Socio-Economic studies.

\section{References}

[1] L. El Guebaly et al, Fus. Eng. Des. 84, 2072 (2009).

[2] S. J. Zinkle, Fus. Eng. Des. 74, 31 (2005).

[3] J. Knaster et al., Nuc. Mats. Energy, Online May 2016

[4] D. Maisonnier. et al, Nuclear Fusion 47, 1524 (2007).

[5] D.J. Ward, Fus. Eng. Des. 82, 528 (2007).

[6] N. Taylor et al, Fus. Eng. Des. 87, 476 (2012).

[7] US Energy Research and Development Administration Report (1976) ERDA-76/110 (later published in Dean, J. Fusion Energy $17,263(1998))$

[8] H. Cabal et al., submitted to Energy Strategy Reviews.

[9] K. Vaillancourt et al., Energy Policy 36, 2296 (2008). 